\title{
A high resolution spectroscopic study of the extraordinary planetary nebula LMC-N66 ${ }^{\star \star \star \star}$
}

\author{
M. Peña ${ }^{1}$, W.-R. Hamann ${ }^{2}$, M. T. Ruiz ${ }^{3}$, A. Peimbert ${ }^{1}$, and M. Peimbert ${ }^{1}$ \\ 1 Instituto de Astronomía, Universidad Nacional Autónoma de México, Apdo. Postal 70-264, 04510 México, DF, México \\ 2 Lehrstuhl Astrophysik der Universität Potsdam, Am Neuen Palais 10, 14469 Potsdam, Germany \\ 3 Depto. de Astronomía, Universidad de Chile, Casilla 36-D, Santiago, Chile
}

Received 16 December 2003 / Accepted 3 February 2004

\begin{abstract}
The planetary nebula N66 in the Large Magellanic Cloud is an extraordinary object, as it is the only confirmed PN where the central star is a Wolf-Rayet star of the nitrogen sequence, i.e. of type [WN]. Moreover, the star showed a dramatic brightness outburst in 1993-1994. In a previous paper (Hamann et al. 2003) we analyzed the changing stellar spectra and found evidence that the central star is most likely a binary system where a white dwarf presently accretes matter from a non-degenerate companion at a high rate. Thus the object is a candidate for a future type Ia supernova in our cosmic neighborhood. In the present paper we analyze the morphology and kinematics of the nebula, using images and high-resolution spectra obtained with the Hubble Space Telescope (HST) and the Very Large Telescope (ESO-VLT). The object presents a complex multipolar structure, dominated by very bright lobes located at both sides of the central star and separated by a narrow waist. In addition there is a pair of very extended and twisted loops, also pointing in opposite directions; their symmetry axis and collimation angle differs from those of the bright lobes. High resolution spectroscopy reveals two main velocity components, "approaching" material at an average heliocentric radial velocity of $V_{\text {rad }}=248 \pm 30 \mathrm{~km} \mathrm{~s}^{-1}$ and similarly bright "receding" material at $V_{\text {rad }}=331 \pm 25 \mathrm{~km} \mathrm{~s}^{-1}$. A systemic velocity of about $300 \mathrm{~km} \mathrm{~s}^{-1}$ is derived. Opposite lobes and loops possess opposite velocities. Furthermore there are knots and filaments of complex structure and kinematics. Close to the central star, nebular gas is found, receding at very high velocity $\left(125 \mathrm{~km} \mathrm{~s}^{-1}\right.$ relative to the system). The morphology and kinematics of LMC-N66 can be explained as the result of episodic bipolar ejections with changing axis. The bipolar structures could have been produced by collimated streams ejected from a precessing central source. We suggest that the precession could have been produced by an external torque, possibly due to a binary companion. Young, fast-moving nebular knots close to the star appear slightly He- and N-richer than the main body of the nebula, but are still hydrogen-rich in contrast to the helium-dominated atmosphere of the [WN]-type central star. In the binary scenario, this nebular matter must have been accreted from the non-degenerate companion and re-ejected before it was fully burnt.
\end{abstract}

Key words. ISM: jets and outflows - ISM: kinematics and dynamics - galaxies: Magellanic Clouds - ISM: planetary nebulae: individual: LMC-N66 - stars: mass-loss - stars: Wolf-Rayet

\section{Introduction}

The extraordinary planetary nebula LMC-N66 (SMP83, WS35, LM1-52) is the only known PN ionized by a [WN] central star (with one possible galactic exception reported recently by Morgan et al. 2003). In 1990, the central star increased its brightness by several magnitudes and developed very prominent WN features caused by a huge mass-loss event. Such an

Send offprint requests to: M. Peña,

e-mail: miriam@astroscu.unam.mx

* Partly based on observations made with the NASA/ESA Hubble Space Telescope obtained at the STScI, which is operated by AURA, Inc., under NASA contract NAS 5-26555.

$\star \star$ Partly based on data collected at the European Southern Observatory, Chile. Proposal ESO 68.C-0149(A). event has been suggested to be the result of a probably very late thermal pulse (Peña et al. 1994) or, alternatively, as due to large atmospheric instabilities (Peña et al. 1997). Presently both suggestions seem to have been discarded (Vassiliadis 1996; Hamann et al. 2003), but the real causes of the stellar outburst are still unknown.

Since 1993 we have followed the stellar changes performing observations with ground-based telescopes and the satellites IUE and HST. Our follow-up has demonstrated that the huge Wolf-Rayet features and the stellar continuum have been slowly fading and, in our last HST spectrum (January 2000) the star showed an emission only slightly higher than that of the pre-outburst epoch. A detailed spectral analysis of the central star spectrum at different epochs has been performed by Hamann et al. (2003), based on models for rapidly expanding 
Table 1. Scientific observations retrieved from HST archives. STIS observations are slitless.

\begin{tabular}{llcrccc}
\hline \hline ID & Observation & Instrument & Grating & $\Delta \lambda(\AA)$ & dd/mm/yy & Exp. (s) \\
\hline 5185 & X25H101T & FOC & F501N & $4973-5047$ & $05 / 02 / 94$ & 1996 \\
9077 & O6EL5Y010 & STIS & MIRVIS & & $28 / 02 / 02$ & 120 \\
$\prime \prime$ & O6EL5Y030 & STIS & G750M & $6295-6867$ & $\prime \prime$ & 240 \\
$\prime \prime$ & O6EL5Y040 & STIS & G430M & $4818-5104$ & $\prime \prime$ & 90 \\
\hline
\end{tabular}

atmospheres. These authors proposed that the outburst did not occur in a single star, but most likely in a binary system undergoing mass exchange which might become a type Ia supernova.

Dopita et al. (1985) found that the nebula N66 possesses extraordinary internal dynamics. From lines that are well split they derived a high expansion velocity of about $2 V_{\exp }=$ $85 \mathrm{~km} \mathrm{~s}^{-1}$ (measured as the peak to peak separation of the blue and red components) and a mean local standard of rest (LSR) radial velocity of $274.5 \mathrm{~km} \mathrm{~s}^{-1}$. Later, Dopita et al. (1993) have argued in favor of a LSR system velocity of $232 \mathrm{~km} \mathrm{~s}^{-1}$. The well split lines represent an evidence of a bipolar flux, instead of a symmetric expanding shell. According to Dopita et al. (1985), the outermost real components spread in velocity over a range of about $225 \mathrm{~km} \mathrm{~s}^{-1}$.

In this work we analyze the morphology and kinematics of the nebula. This should shed more light on the evolutionary origin of this exceptional object and its possibly even more exciting future as a SN Ia candidate. One objective is to look for further indications of the binary nature of the N66 nucleus.

In the following, we first discuss spatially resolved images (Sect. 2) and slitless spectroscopy (Sect. 3) of N66 retrieved from the archives of the Hubble Space Telescope (HST). In Sect. 4 we present new data which we obtained with the Ultraviolet Echelle Spectrograph (UVES) at the Very Large Telescope of the European Southern Observatory (ESO), and long-slit spectra which were taken with the Space Telescope Imaging Spectrograph (STIS) on board the HST. Section 5 is devoted to the nebular analysis, and Sect. 6 summarizes the discussion and conclusions.

\section{HST images: The nebular morphology}

Several narrow-band direct images of N66, with high spatial resolution, are available at HST archives. Some of them have been used previously to analyze the morphology of the ionized gas. A thorough description of some of the images and a previous discussions on the morphology of N66 can be found in Blades et al. (1992), Dopita et al. (1993), Vassiliadis (1996) and Vassiliadis et al. (1998).

For this work we have retrieved from HST public archives the FOC image X25H101T obtained through the filter F501N (such a filter has a maximum transmission at $\lambda=5010 \AA$ and a FWHM of $74 \AA$, isolating therefore the strong [O III] 5007 nebular emission line) and some recent STIS images and slitless spectroscopy obtained for N66 as part of an extensive program for studying the morphology and other nebular parameters of the Magellanic Cloud PNe (Stanghellini et al. 2002; Shaw et al., in prep.). The characteristics of the retrieved images are listed in Table 1.

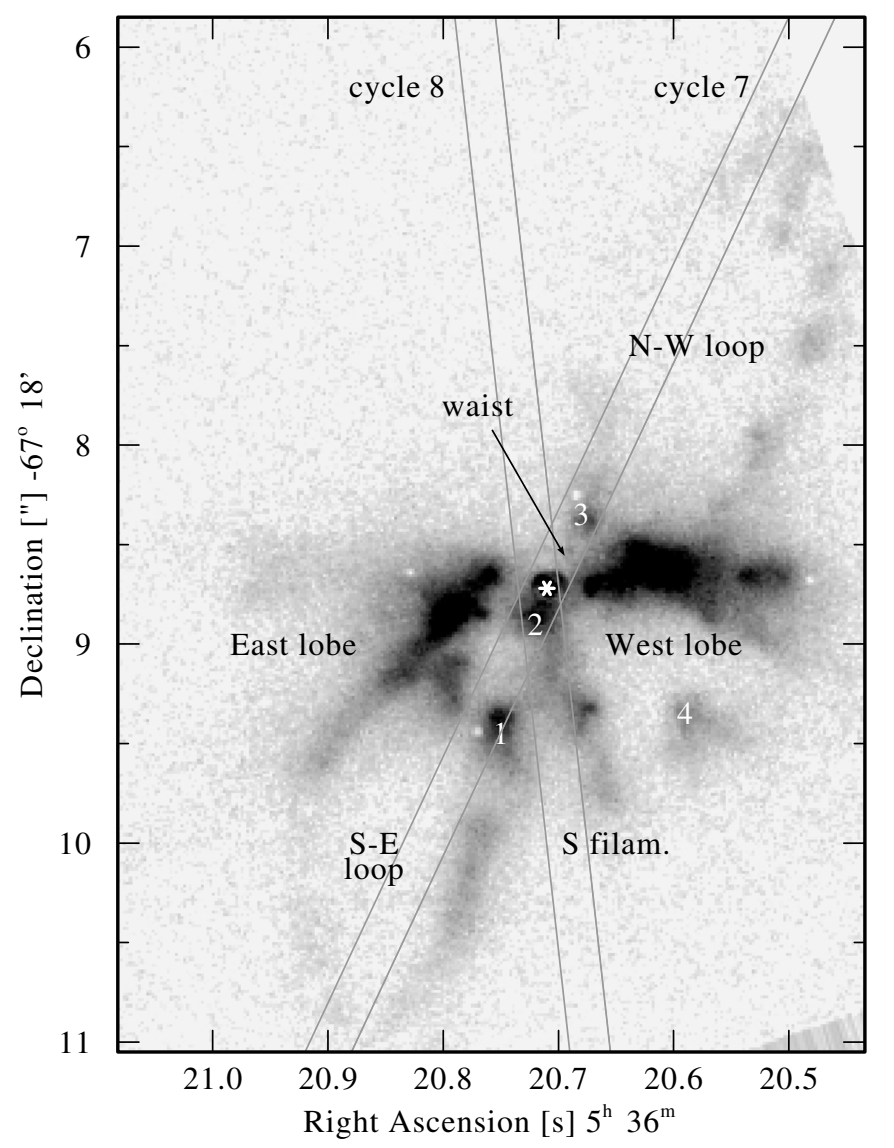

Fig. 1. Direct image X25H101T, obtained with the HST WFPC and a $F 501 N$ filter. The image has been rotated to present North at top and East at left. The bright East and West lobes and two faint loops (in the South-East and North-West directions) are noticeable. The numbers 1, 2, 3 and 4 mark nebular knots studied in the text. The slit orientations of our two HST STIS spectra are also indicated.

The FOC image is shown in Fig. 1 where some important knots and filaments have been marked and we have superimposed two sets of parallel lines showing the position of the STIS long slits used in Cycles 7 and 8 for our spectroscopic observations (see Sect. 4). The STIS images are shown in Fig. 2 and will be discussed in more detail in the next section.

LMC-N66 appears as an extended nebula with a very complex morphology. The central star is clearly seen in the center of two bright lobes almost aligned in the E-W direction, and separated by a narrow waist. Several knots and long filaments, mainly directed to the south, are also prominent. The extension of each lobe amounts to approximately $0.3^{\prime \prime}$ by $1.2^{\prime \prime}$, which represents a projected physical dimension of 0.07 by $0.28 \mathrm{pc}$, if a distance modulus of 18.45 is assumed for the LMC (Feast 

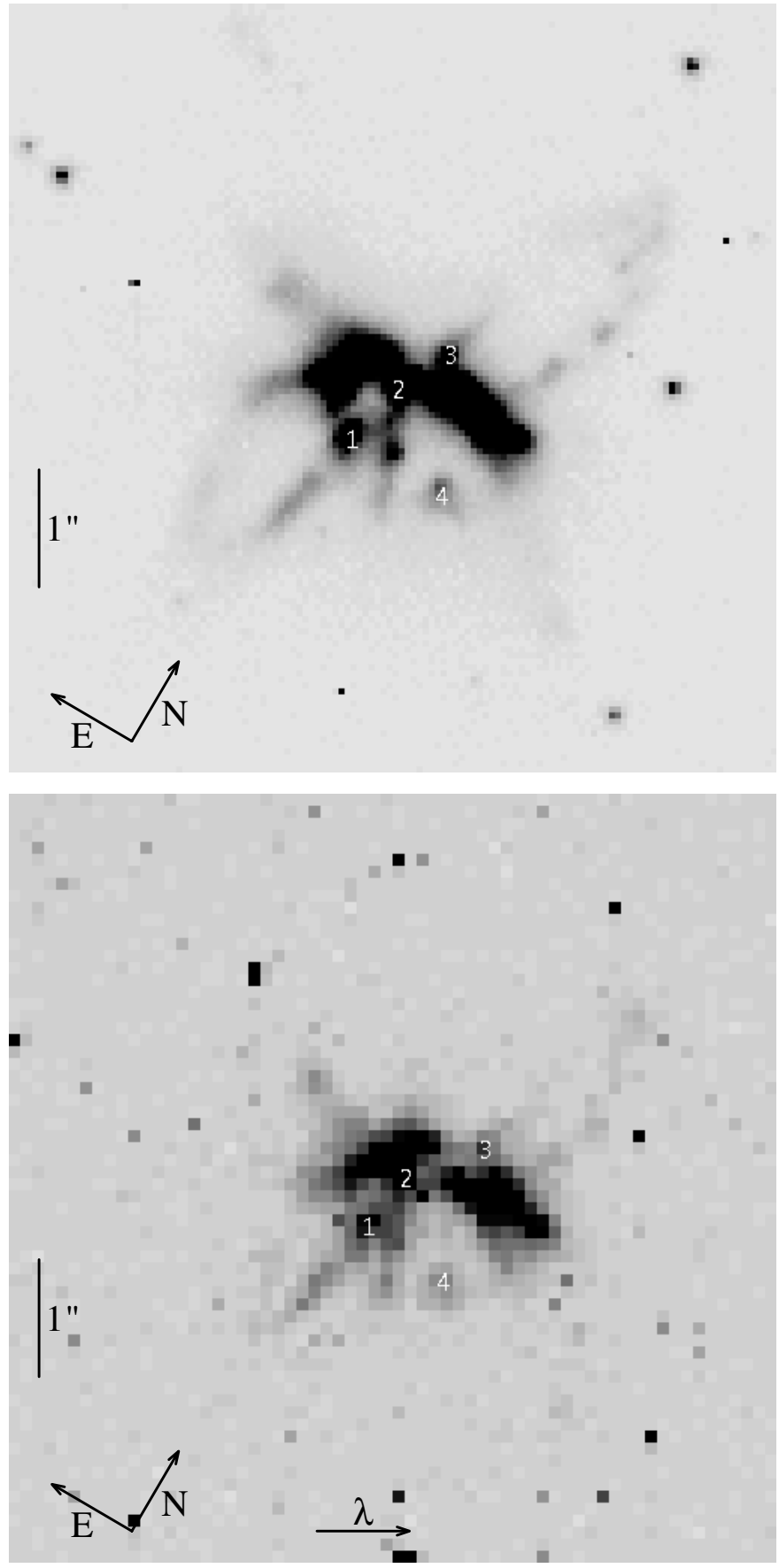

Fig. 2. The STIS-MIRVIS image (top) is compared to STIS slitless [O III] 5007 spectrum (bottom). Both images are centered at knot 2 and have the same orientation (top at PA $30.39^{\circ}$ ) and same spatial resolution. There is an evident deformation of N66 in the slitless spectrum, due to the differences in radial velocity of the structures.

1988; the most recent Cepheid calibration by Benedict et al. 2002 confirms this value). The lobes are not single structures, the western one is formed by at least two very close filaments of about equal extension which show approaching and receding velocities, as will be shown in the kinematical analysis (Sect. 3). Something similar holds for the eastern lobe. Thus, the lobes correspond to two opposite ejections forming by a bipolar structure, with a very narrow opening angle.
Very interesting structures are the couple of faint, narrow and bent filaments which seem to start near the star, extend along the bright lobes, and bend towards the S-E and N-W directions, respectively (they have been marked as "N-W loop" and "S-E loop" in Fig. 1). These filaments (in particular the $\mathrm{S}-\mathrm{E}$ one) seem to close in the form of long loops. The projected dimensions of these loops are about $1^{\prime \prime}$ wide by $2.4^{\prime \prime}$ long (corresponding to about 0.23 by $0.57 \mathrm{pc}$ ). They give a nice twisted "butterfly" appearance to the nebula. The loops open in a projected angle of about $40^{\circ}$. These loops represent clearly another bipolar structure, with a different orientation than that of the main lobes and with a different degree of collimation. The northwest loop is formed by a series of knots distributed in an arc.

Searching carefully, one finds that almost each structure in either west or east zone has a counterpart on the other side of the central star: the east and west lobes are mutual counterparts, and the twisted N-W loop has its counterpart in the also bent E-S loop. A very faint emission in the S-W quadrant at $2.5^{\prime \prime}$ from the central star (at a position angle, counted from north over east, of $\mathrm{PA} \sim 240^{\circ}$ ) seems to have an also very faint counterpart in the N-E quadrant (see Fig. 2). The only structures without evident counterparts are the southern knot 2 and its associated jet-like filament which extends over about $1^{\prime \prime}$, and the also southern knot 4. But basically N66 displays two bipolar structures and jet-like ejections, at different position angles and with varying degree of collimation, suggesting a history of episodic ejections along a changing axis.

The narrow waist is oriented at $\mathrm{PA} \sim 45^{\circ}$. No emission is detected in a wide zone of the N-E quadrant while in the S-W quadrant only the faint knot 4 is found. Blades et al. (1992) suggested that this non-emitting zone could be due to a dusty toroid similar to the ones found around the central stars in some "butterfly" shaped nebulae, like the galactic PN NGC 6302. In the case of LMC-N66, such a dusty toroid would be centered at the central star and would have impeded the expansion of the nebular gas in the NE-SW directions. However the star is not obscured by dark material, in contrast to the case of NGC 6302, and therefore Blades et al. (1992) suggest the possibility that the disk around the N66 central star is now almost dissipated.

A large amount of cold dust indeed exists in N66, as deduced from IRAS detection. Schwering (1989) reports $F(12 \mu) \leq 0.15 \mathrm{Jy}, F(25 \mu) \leq 0.11 \mathrm{Jy}, F(60 \mu)=1.2 \mathrm{Jy}$, and $F(100 \mu)=6.2$ Jy for LMC-N66. However, this dust is apparently not concentrated in the zone of the waist, as deduced from the fact that the receding material does not appear much more extinct nor more reddened than the approaching gas. The values for $\mathrm{c}(\mathrm{H} \beta)$ for both, approaching and receding components, presented in Sect. 4, are equal within uncertainties. The same holds for the gas immediately behind the central star. In Sect. 5 we will show that knot 3 (which is very redshifted) does not present particularly larger reddening than other nebular zones.

Recently, several authors have proposed other mechanisms for producing a narrow waist and collimated jets and filaments in planetary nebulae. The main ones are:

- the interaction of jets or collimated fast winds - produced in binary evolved stars which have developed a density 




Fig. 3. Sketch of the suggested three-dimensional structure of the nebula N66. The little asterisk refers to the position of the central star. The knots are marked by their number, and other structures are also labelled (cf. Figs. 1 and 2).

equatorial enhancement due to mass transfer - with a previous, slow AGB wind (Soker 2002);

- magnetic collimation of the fast post-AGB winds which can produce a variety of bipolar structures as well as jets and ansae (García-Segura 1997; García-Segura \& López 2000). In this scenario, rotating (twisted) jets and point-symmetric structures require magnetic collimation around a precessing source or a magnetic collimation axis misaligned with respect to the symmetry axis of the bipolar outflow.

Considering the multipolar, highly-collimated morphology and extraordinary kinematics of N66, the latter possibility is the one we prefer most. But in either case, both scenarios involve a precessing source from which the bipolar ejections emerge with different orientations. According to García-Segura (1997), the knotty appearance of the filaments and loops could be due to "kink" instabilities and not to episodic ejections.

\section{Slitless spectroscopy}

The direct image O6EL5Y010 (STIS-MIRVIS), and the slitless spectroscopic image O6EL5Y040 in the [O III] 5007 emission line are compared in Fig. 2. We have not included the image O6EL5Y030 (6295-6867 $\AA$ ) because H $\alpha$ and the [N II] 6548 and 6583 lines are very overlapped. But we have checked that the [N II] lines yield a similar picture as [O III] 5007.

In Fig. 2 the images have been centered at knot 2 (the nearest to the central star) and the spatial resolution is the same for both. Although the slitless spectra are lacking the adequate spectral resolution, a rough analysis of the kinematical behavior of the ionized gas can be carried out.
By using blinking techniques, we have compared the MIRVIS and the slitless [O III] 5007 spectrum and found that:

- the north part of the nebula (including the north zone of the both lobes and knot 3 ) and the E-S loop are mainly shifted to the red, while the southern knots and filaments and the N$\mathrm{W}$ loop are mainly shifted to the blue. The most redshifted feature is knot 3 which, according to our high resolution STIS spectroscopy (see Sect. 4.3) has a heliocentric radial velocity of about $425 \mathrm{~km} \mathrm{~s}^{-1}$;

- the west lobe consists of two filaments (projected approximately parallel and of about the same length) showing different radial velocities. The southern component is the bluer. Our interpretation is that these two filaments are oriented in such a way that the southern filament is approaching while the other is receding (see Fig. 3). The filaments do not close;

- the east lobe also presents components at different velocities. The northern zone of this lobe is very redshifted. The southern zone shows a lower velocity than knot 2 (central knot). As in the west lobe, these filaments do not close;

- the loops show opposite velocities: the N-W loop is blueshifted (at least its western filament) and the E-S loop is redshifted. The backward filaments are very redshifted in both loops;

- knot 4 in the S-W quadrant has a radial velocity similar to knot 2.

The suggested three-dimensional structure of the nebula is sketched in Fig. 3. It is based on the assumption that the nebula is expanding radially from the central star. Hence those knots or filaments which are moving towards the observer (relative to the systemic velocity) are located in front of the plane-of-sky, while receding structures are located in the back hemisphere. Note that the gas distribution is very filamentary and not spherically symmetric. There is almost no gas in a large zone to the north of the main lobes.

\section{Low and high-resolution spectroscopic data}

We performed ground-based high-resolution spectroscopy with the Ultraviolet Echelle Spectrograph (UVES, D'Odorico et al. 2000) at the ESO VLT Kueyen Telescope in Chile. These observations cover the whole nebula and, therefore, allow to deduce the global physical conditions and nebular kinematics (see Sect. 4.1). We also obtained spatially resolved HST STIS longslit spectra of low and high resolution in two consecutive years (Cycles 7 and 8). With the latter observations, we isolated some particular knots and filaments near the central star, for which we have derived the kinematical behavior and the physical conditions (Sects. 4.2 and 4.3).

\subsection{ESO VLT UVES observations}

VLT UVES high resolution spectroscopy was performed in March 12, 2002 (UT). The red and blue arms were used simultaneously in two settings to cover a wide spectral range. The slit was set to a width of $3^{\prime \prime}$ and oriented east-west. 
The slit height was $10^{\prime \prime}$ in the blue arm and $12^{\prime \prime}$ in the red one. The atmospheric dispersion corrector was used to keep the same observed region within the slit regardless of the air mass.

Several frames were obtained in different spectral ranges covering the wavelength range from 3100 to $9500 \AA$. The exposure times for the frames vary from 300 to $600 \mathrm{~s}$, trying to obtain a good signal to noise for the faint lines without saturating the bright ones. The frames with the same spectral ranges were combined to improve the signal-to-noise and to eliminate cosmic rays. The $F W H M$ resolution of the spectra is better than $\Delta \lambda / \lambda \sim 12000$, which allows us to examine the velocity field of the nebula with a precision better than $25 \mathrm{~km} \mathrm{~s}^{-1}$. The spectra were reduced using $\mathrm{IRAF}^{1}$ echelle reduction package, following the standard procedure of bias subtraction, aperture extraction, flatfielding, wavelength calibration and flux calibration. For flux calibration the standard star EG 247 was observed. Calibrated spectra were analyzed to determine the physical conditions and the kinematics in N66.

All the emission lines detected in these spectra are of nebular origin and present a very well separated double profile, completely similar to the ones reported by Dopita et al. (1985). These split profiles definitely confirm the bipolar structure of N66. The emission is dominated by the bright lobes shown in Fig. 1. Emission from the central star was not detected due to its faintness and the high dispersion employed.

Figure 4 shows the calibrated profile extracted for the [O III] $5007 \AA$ line. The two-components structure detected in this line is similar for all nebular lines. We found that both, the blue and red components, are very wide but well separated. Taking the system heliocentric velocity as the velocity of the minimum between the red and blue components we find $V_{\text {sys }} \sim 300 \pm 20 \mathrm{~km} \mathrm{~s}^{-1}$, which is considerably redshifted for a PN in the LMC. Dopita et al. (1993) have argued against adopting the average of the red and blue components as the system velocity and suggested a $V_{\text {sys }} \sim 232.5 \mathrm{~km} \mathrm{~s}^{-1}$, but we found no support for such a suggestion.

The mean heliocentric radial velocities of the receding and approaching components are $248 \mathrm{~km} \mathrm{~s}^{-1}$ and $331 \mathrm{~km} \mathrm{~s}^{-1}$, respectively. This difference in velocities corresponds very well to the $85 \mathrm{~km} \mathrm{~s}^{-1}$ separation measured by Dopita et al. (1985). However this difference cannot be interpreted as a classical expansion velocity of the nebula, because the line profiles (in particular the blue side) show a complex structure due to a mixture of components at different velocities.

The blue line spans over a range of $160 \mathrm{~km} \mathrm{~s}^{-1}$ at FWZI and can be easily (and confidently) deblended in two Gaussian components at velocities $240 \mathrm{~km} \mathrm{~s}^{-1}\left(F W H M \sim 22 \mathrm{~km} \mathrm{~s}^{-1}\right)$ and $270 \mathrm{~km} \mathrm{~s}^{-1}\left(F W H M \sim 12 \mathrm{~km} \mathrm{~s}^{-1}\right)$, leaving no residual. The red line also shows a mixture of components. At FWZI it spans over $130 \mathrm{~km} \mathrm{~s}^{-1}$. A fit with two Gaussian components at $331 \mathrm{~km} \mathrm{~s}^{-1}\left(F W H M \sim 23 \mathrm{~km} \mathrm{~s}^{-1}\right)$, and $373 \mathrm{~km} \mathrm{~s}^{-1}(F W H M \sim$ $25 \mathrm{~km} \mathrm{~s}^{-1}$ ) reproduces fine the profile, with no residuum left. The deblend for the blue and red profiles is shown in Fig. 4.

1 IRAF is distributed by NOAO, which is operated by AURA, under cooperative agreement with the NSF.
Table 2. Observed Balmer decrement and reddening derived from VLT UVES data.

\begin{tabular}{lcc}
\hline \hline Line ratio & Approach. & Receding \\
\hline $\mathrm{H} \alpha / \mathrm{H} \beta$ & 3.321 & 3.416 \\
$\mathrm{H} \gamma / \mathrm{H} \beta$ & 0.450 & 0.462 \\
$\mathrm{H} \delta / \mathrm{H} \beta$ & 0.239 & 0.241 \\
$\mathrm{H} 7 / \mathrm{H} \beta$ & 0.151 & 0.141 \\
$\mathrm{H} 9 / \mathrm{H} \beta$ & 0.061 & 0.056 \\
\hline$c(\mathrm{H} \beta)$ & $0.23 \pm 0.05$ & $0.27 \pm 0.05$ \\
\hline
\end{tabular}

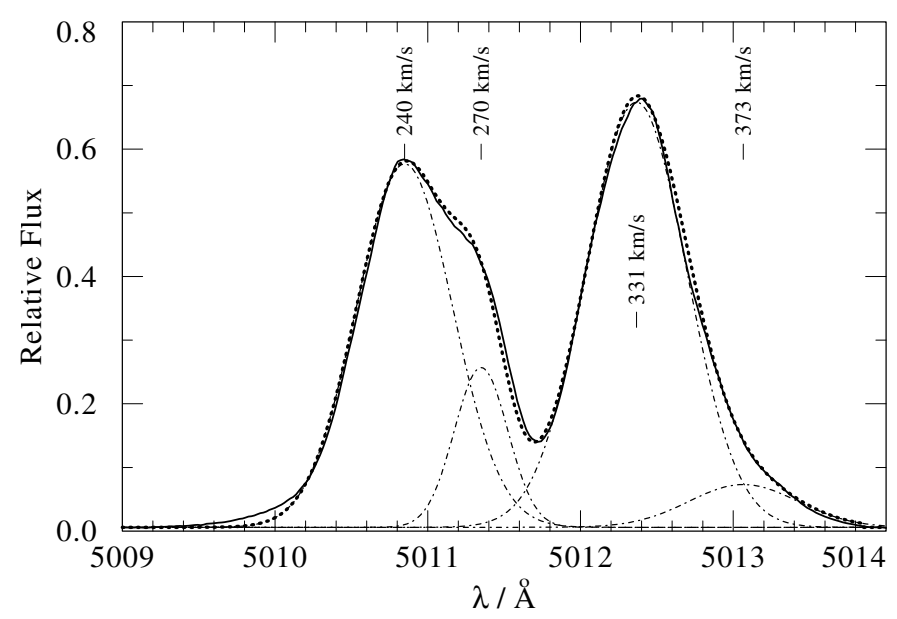

Fig. 4. High spectral-resolution emission of [O III] $5007 \AA$ line, obtained with VLT UVES (solid line). It shows the approaching and receding gas. The dotted line is a fit obtained by adding four Gaussians, which are also shown individually. The approaching emission is a blend of at least two components at radial velocities of 240 and $270 \mathrm{~km} \mathrm{~s}^{-1}$. The red side is also complex, presenting a bright maximum at $331 \mathrm{~km} \mathrm{~s}^{-1}$ and a faint component at $373 \mathrm{~km} \mathrm{~s}^{-1}$ (see discussion in the text).

By comparing the above results with the slitless spectroscopy, we deduce that the blueshifted components are emitted by the southern zones of the main lobes and the southern knots and filaments, while the redshifted emissions are produced mainly in the northern parts of the nebula.

In Table 2 we list the observed Balmer line ratios and the logarithmic reddening corrections, $c(\mathrm{H} \beta)$, as derived from the Balmer decrement for the blue and red emissions. The theoretical recombination line ratios by Storey \& Hummer (1995) and the reddening law by Seaton (1979) were used. We found that reddening is similar (within uncertainties) in both line components. This indicates that the red zones are not more extinct than the blue ones, in contrast to what would be expected if they were obscured by a dusty torus forming the nebular waist. Hamann et al. (2003) deduced $E(B-V) \sim 0.16$ for the central star (which is equivalent to $c(\mathrm{H} \beta) \sim 0.24$ ), which shows that the central star is also not affected by a larger reddening.

Fluxes for the most important emission lines, detected from the approaching and receding zones of the nebula, are compiled in Table 4 and will be used in Sect. 5 for the plasma diagnostics. 
Table 3. Log of scientific HST STIS long-slit observations.

\begin{tabular}{llcrcccc}
\hline \hline ID & Observation & Detector & PA $\left(^{\circ}\right)$ & Grating & $\Delta \lambda(\AA)$ & dd/mm/yy & Exp. $(\mathrm{s})$ \\
\hline 7482 & o55r01010 & FUV-MAMA & -20.789 & G140L & $1140-1730$ & $08 / 01 / 99$ & 4500 \\
$\prime \prime$ & o55r01020 & NUV-MAMA & -20.789 & G230L & $1568-3184$ & $"$ & 2880 \\
$\prime \prime$ & o55r01030 & CCD & -20.733 & G430L & $2900-5700$ & $"$ & 1680 \\
$\prime \prime$ & o55r02010 & CCD & -20.733 & G430M & $4563-4849$ & $\prime$ & 1320 \\
$\prime \prime$ & o55r02020 & CCD & -20.733 & G750L & $5236-10266$ & $"$ & 2280 \\
8462 & o5im01010 & FUV-MAMA & 5.632 & G140L & $1140-1730$ & $04 / 02 / 00$ & 6000 \\
$\prime \prime$ & o5im01020 & NUV-MAMA & 5.632 & G230L & $1568-3184$ & $"$ & 3660 \\
$\prime \prime$ & o5im02010 & CCD & 5.609 & G430L & $2900-5700$ & $"$ & 2040 \\
$\prime \prime$ & o5im02020 & CCD & 5.609 & G430M & $4818-5104$ & $"$ & 2100 \\
\hline
\end{tabular}

\subsection{HST STIS long-slit observations}

Two sets of STIS long-slit observations (including low- and high-spectral resolution data) were obtained by us during Cycles 7 and 8 (ID programs 7482 and 8462). For both cycles the slit dimensions were $52^{\prime \prime} \times 0.2^{\prime \prime}$, centered at the central star. MAMA and CCD detectors were employed to cover the widest possible spectral range (from UV to visible). Spectra were calibrated with the standard STIS pipeline, version 2.0 (released in October 1998) for Cycle 7, and version 2.3 (released in September 1999) for Cycle 8. The log of observations is presented in Table 3.

Long slit observations with STIS simultaneously provide stellar and nebular spectra. As mentioned in the introduction, a thorough stellar analysis has been presented by Hamann et al. (2003). In the following we discuss the spectra of several bright nebular knots, detected at different distances from the central star.

The slit orientations of both STIS observations are indicated in Fig. 1. In Cycle 7 the three bright knots, marked 1, 2 and 3 in the figure, were covered by the slit oriented at PA $-20.7^{\circ}$. When in Cycle 8 the slit was oriented at PA $\sim+5.6^{\circ}$, we reobserved knot 2 (the nearest to the central star) and part of the filament extending from knot 2 to the south.

In the next subsection we discuss the kinematical behavior of these knots and filaments.

\subsection{STIS high resolution spectra}

In Cycle 7, the high-resolution grid G430M (centered at $4706 \AA$ ) was used covering the spectral range 4563-4849 with a resolution of about $0.28 \AA$ per pixel. The strongest emission line in such an interval is He II 4686. A contour map (position versus wavelength) of this emission is shown in Fig. 5c. The emission is clearly bipolar: the southern knots 1 and 2 present heliocentric radial velocities of 330 and $309 \mathrm{~km} \mathrm{~s}^{-1}$, respectively, while knot 3, at north, is strongly redshifted with $V_{\text {rad }}=425 \mathrm{~km} \mathrm{~s}^{-1}$.

In Cycle 8 the same grid, centered at $4961 \AA$, was employed covering the spectral range from 4818 to $5104 \AA$. Therefore $\mathrm{H} \beta$, and [O III] 4959 and 5007 were observed for knot 2 and its associated filament, which extends for $1^{\prime \prime}$ to the South-West. Figures $5 \mathrm{a}$ and $5 \mathrm{~b}$ show the contours of the structures. The kinematics observed in Cycle 7 is confirmed by the Cycle 8 observations: knot 2 shows $V_{\text {rad }}=309 \mathrm{~km} \mathrm{~s}^{-1}$, and the filament has about the same velocity.

In addition, we have detected a faint red component extending from the central star to the north, with a velocity of about $425 \mathrm{~km} \mathrm{~s}^{-1}$ (similar to the velocity of knot 3), and a very faint knot in the South (about 1" from the central star) moving at $450 \mathrm{~km} \mathrm{~s}^{-1}$

Adopting that the heliocentric radial velocity of the system is about $300 \mathrm{~km} \mathrm{~s}^{-1}$ (as deduced from VLT UVES data), we find that knot 2 and its filament are at systemic velocity and therefore in the plane of the sky, while knots 1 and 3 are both receding with projected radial velocities of about +30 and $+125 \mathrm{~km} \mathrm{~s}^{-1}$, respectively.

In conclusion, high spectral resolution data from both cycles show the following kinematical behavior for the observed material: the faint nebular gas to the north of the central star is receding at more than $100 \mathrm{~km} \mathrm{~s}^{-1}$, relative to the system, while the central knot 2 and its southern filament are at $V_{\text {sys }}$ and knot 1 is slightly redder $\left(+30 \mathrm{~km} \mathrm{~s}^{-1}\right)$.

\section{STIS low-resolution data and UVES line fluxes: Nebular conditions in the observed knots}

The wide spectral range obtained with STIS provides many important plasma diagnostic line ratios and emission lines useful for determining the ionization structure and chemical composition of the observed knots. For this aim, we extracted three nebular spectra corresponding to knots 1, 2 and 3 from STIS Cycle 7 low-resolution spectra.

The observed (not corrected for reddening) UV and optical emission line fluxes, relative to He II $1640 \AA$ and $\mathrm{H} \beta$ respectively, are presented in Table 4 , where we have also included the line fluxes measured before the outburst and the flux ratios derived for each component from VLT UVES observations. One should be aware that the emission from the knots is much fainter that the global emission of the nebula. Therefore the flux ratios for the knots are more uncertain, and the physical parameters and ionic abundances have large errors. Nevertheless these data allow for a rough analysis of the chemical abundances near the central star.

In our previous papers we found that the nebular lines have not changed during the stellar outburst. This is corroborated by 


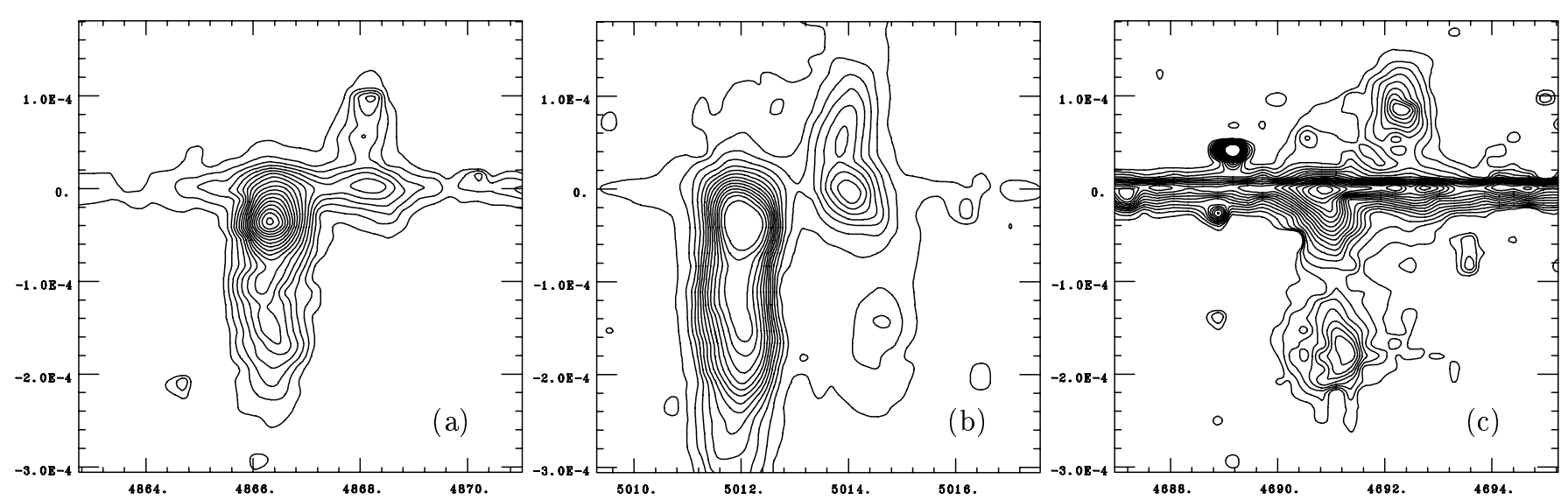

Fig. 5. Contours of flux from long-slit spectra taken with HST STIS, showing in high resolution a) H $\beta$ and b) [O III] 5007, observed in Cycle 8, and c) He II 4686 (observed in Cycle 7). The abscissa gives the spatial position in degrees, relative to the central star. The orientations of the slit are indicated in Fig. 1. The kinematical structure of different knots is clearly noticeable (see discussion in the text). In the case of He II 4686 the wide stellar emission line is detected. The very intense spots are imperfectly removed cosmic rays.

Table 4. Observed nebular (reddened) line flux ratios.

\begin{tabular}{|c|c|c|c|c|c|c|c|}
\hline \multirow[t]{2}{*}{$\lambda(\AA)$} & \multirow[t]{2}{*}{ Ion } & \multicolumn{6}{|c|}{$-F_{\lambda} / F(\mathrm{H} \beta)^{1}$} \\
\hline & & Pre-outb. ${ }^{2}$ & Approach. ${ }^{3}$ & Receding $^{3}$ & Knot 1 & Knot 3 & Knot 2 \\
\hline 1240 & $\mathrm{NV}$ & 1.83 & - & - & 0.92 & 1.39 & 1.54 \\
\hline 1404 & $\mathrm{O} I V+\mathrm{Si} I V$ & 0.43 & - & - & $0.45:$ & 0.50 : & - \\
\hline 1488 & N IV & 0.94 & - & - & 0.94 & 0.99 & 1.01 \\
\hline 1550 & CIV & 1.32 & - & - & 1.03 & 1.14 & 0.90 \\
\hline 1640 & He II & 2.69 & - & - & 2.24 & 2.48 & 2.40 \\
\hline 1666 & O III] & 0.30 & - & - & 0.22 & 0.30: & - \\
\hline 1750 & N III] & 0.43 & - & - & 0.45 & - & - \\
\hline 1909 & $\left.\mathrm{C}_{\mathrm{III}}\right]$ & 0.43 & - & - & 0.51 & 0.45: & 0.38 : \\
\hline 2427 & [Ne IV] & 1.16 & - & - & 0.90 & 1.09 & 0.48 : \\
\hline 3349 & $\mathrm{Ne} \mathrm{V}$ & - & 0.37 & 0.34 & - & 0.37 & 0.22 \\
\hline 3430 & {$[\mathrm{Ne} \mathrm{V}]$} & 1.10 & 1.00 & 0.88 & 0.88 & 1.12 & 1.01 \\
\hline 3727 & {$[\mathrm{O} \mathrm{II}]$} & 0.86 & 1.05 & 0.91 & 0.93 & 0.54 & 0.70 \\
\hline 3869 & [Ne III] & 0.91 & 0.89 & 0.92 & 0.94 & 0.70 & 1.23 \\
\hline 4070 & {$[\mathrm{~S} \mathrm{II}]$} & 0.041 & 0.035 & 0.032 & - & - & - \\
\hline 4363 & [O III] & 0.20 & 0.23 & 0.22 & 0.20 & 0.18 & 0.21 \\
\hline 4686 & He II & 0.68 & 0.69 & 0.59 & 0.51 & 0.74 & 0.59 \\
\hline 4711 & [Ar IV] & 0.12 & 0.09 & 0.10 & 0.08 & - & 0.12 : \\
\hline 4725 & [Ne IV & 0.02 & - & - & 0.03 & - & - \\
\hline 4740 & [Ar IV] & 0.09 & 0.08 & 0.09 & 0.08 & - & 0.10 : \\
\hline 5007 & [O III] & 9.25 & 9.11 & 9.02 & 10.20 & 8.10 & 9.64 \\
\hline 5755 & {$[\mathrm{~N}$ II $]$} & 0.034 & 0.049 & 0.043 & - & - & - \\
\hline 5876 & $\mathrm{He} \mathrm{I}$ & 0.09 & 0.09 & 0.10 & - & - & 0.12 \\
\hline 6312 & [S III $]$ & 0.03 & 0.05 & 0.04 & 0.03: & - & - \\
\hline 6563 & $\mathrm{H} \alpha$ & 3.14 & 3.32 & 3.42 & 3.08 & 3.30 & 3.13 \\
\hline 6583 & [N II] & 1.30 & 2.20 & 1.91 & 1.33 & 0.90 & 1.37 \\
\hline 6725 & [S II] & 0.17 & 0.13 & 0.14 & 0.09 : & 0.08 : & 0.13 \\
\hline 9079 & [S III] & - & 0.32 & 0.20 & 0.22 & - & - \\
\hline
\end{tabular}

${ }^{1}$ Uncertainties in line fluxes are better than $20 \%$, except for those lines marked with a colon.

2 pre-outburst data from Peña et al. (1995).

${ }^{3}$ Blue- and redshifted line components from our ESO VLT UVES observations.

the observations presented here. Approaching and receding gas detected with ESO VLT observations show, on the average, the same flux ratios as in the pre-outburst epoch. The differences are minor: the approaching gas presents a slightly higher ionization degree and higher density than the receding material, but on the average, the line ratios are equal within uncertainties to the pre-outburst epoch. Knots 1,2 and 3 also show some small differences among them, all of which can be attributed to differences in the ionization degree, caused by the ionization structure in the nebula: knot 3 presents the highest 
ionization degree (with He II 4686 / $\mathrm{H} \beta \sim 0.74$ ), while knot 1 , with He II 4686 / $\mathrm{H} \beta \sim 0.51$, presents the lowest. Taking an average, the line intensities in the knots, relative to $\mathrm{H} \beta$, are equal, within the uncertainties, to the pre-outburst epoch.

In conclusion, line flux ratios have remained unchanged. The outburst of the central star, which lasted near 10 years, should have reduced drastically the He II Zanstra temperature (Peña et al. 1997; Hamann et al. 2003). But this has not been noticed so far by the nebula, whose ionization degree has not changed. This is consistent with the nebular density of about $2000 \mathrm{~cm}^{-3}$, which implies a recombination time for the nebula of about 50 years.

An uncertain logarithmic reddening correction, $c(\mathrm{H} \beta)$ 0.14 , is derived from STIS data for the knots, in agreement with results from other observations. Knot 3 presents the same reddening than other zones. This implies that the receding material near the central star has a similar reddening than the approaching southern gas.

The observed line fluxes presented in Table 4 have been dereddened using the reddening law by Seaton (1979) and the value of $c(\mathrm{H} \beta)$ obtained for their zone. From the reddening corrected flux ratios we derived diagnostic line ratios and physical parameters (electron temperature $T_{\mathrm{e}}$, and density $n_{\mathrm{e}}$ ), as well as ionic and total abundances, in a consistent manner. The results are listed in Table 5. Electron temperatures of the red-and blue-shifted components resolved in the VLT spectra are equal, and similar within uncertainties to the values obtained from the pre-outburst data. The density, however, is lower in the receding gas.

At first approximation, N66 presents the same chemical composition for all the analyzed zones. All the zones are $\mathrm{H}$-rich and, within uncertainties, we could not detect variations of the oxygen abundance with distance from the central star. The $\mathrm{O} / \mathrm{H}$ abundance ratio (by number, after correcting for the unobserved ions according to the ionization correction factor (ICF) scheme by Kingsburgh \& Barlow 1994) is equal to $(1.4 \pm 0.1) 10^{-4}$.

Helium and nitrogen, however, tend to be slightly enriched in the young knots. The $\mathrm{He} / \mathrm{H}$ number ratio (assuming that $\mathrm{He}=\mathrm{He}^{+}+\mathrm{He}^{++}$) is $0.119 \pm 0.006$ when averaged over the nebula, while marginally higher values are derived for the knots. The same is found for the N/O abundance ratio (by assuming that $\mathrm{N} / \mathrm{O}=\mathrm{N}^{+} / \mathrm{O}^{+}$): knots 2 and 3 (the nearest to the central star) seem slightly richer in $\mathrm{N}(\mathrm{N} / \mathrm{H} \sim 1.2)$ than the global values $(\mathrm{N} / \mathrm{H} \sim 0.85)$.

\section{Discussion and conclusions}

From HST spatially resolved images and high resolution spectroscopy obtained with HST STIS and ESO VLT UVES spectrographs, we have found that N66 shows a complex multipolar morphology with a very narrow waist. Two bright lobes, at opposite sides of the central star, constitute the main body of the nebula. Each lobe presents approaching and receding gas. The lobes are the product of a bipolar ejection oriented more or less in E-W direction.

Particularly important for understanding the morphology of N66 are the two very extended twisted loops in the S-E and
$\mathrm{N}-\mathrm{W}$ quadrant, with dimensions of $1^{\prime \prime}$ by $2.4^{\prime \prime}(0.23$ by $0.57 \mathrm{pc}$ at the distance of the LMC). Both loops open in an angle of about $40^{\circ}$. The projected symmetry axis of the loops is oriented at about $135^{\circ}$ (east from north). STIS slitless spectroscopy shows roughly that the N-W loop is approaching while the $\mathrm{S}$ E loop is receding. The loops are counterpart one of the other, thus, they represent another bipolar structure with a different orientation than the one of the main lobes.

Therefore, outflows in different directions have emerged from the core of N66. At least two of them present bipolar structures. Another jet-like ejection is observed which is associated with knot 2 and directed towards the south. The morphology of N66 demonstrates the existence of an efficient and changing collimating mechanism. There is no evidence that the collimated outflows had been focussed by a dusty ring around the central star. We consider that the multipolar structures have been produced by magnetic collimation of the wind ejected from a precessing source.

The multipolar morphology is also found in the kinematics of N66. VLT UVES spectroscopic data show well split approaching and receding components. The approaching material presents at least two components at $240 \pm 11 \mathrm{~km} \mathrm{~s}^{-1}$ and $270 \pm 6 \mathrm{~km} \mathrm{~s}^{-1}$, with an average velocity of $248 \mathrm{~km} \mathrm{~s}^{-1}$. The receding gas shows an average velocity of $331 \mathrm{~km} \mathrm{~s}^{-1}$, although it also contains several components which spread in a velocity range from 300 to more than $420 \mathrm{~km} \mathrm{~s}^{-1}$. The systemic radial velocity of $\mathrm{N} 66$ is $300 \mathrm{~km} \mathrm{~s}^{-1}$, measured at the minimum between the approaching and receding gas.

High resolution STIS spectroscopy indicates the presence of high velocity gas to the North of the central star, receding at heliocentric radial velocity of $425 \mathrm{~km} \mathrm{~s}^{-1}\left(+125 \mathrm{~km} \mathrm{~s}^{-1}\right.$ relative to the system velocity).

The large differences in velocity between the receding and approaching zones of N66, and its complex kinematics, are similar to those found in the galactic multipolar PNe NGC 2440, NGC 6302 and Mz 3 (see López et al. 1998 and references therein). Some PNe defined as quadrupolar by Manchado et al. (1996) also show similar morphology and kinematical behavior. These latter objects are characterized as possessing a narrow waist and two pairs of lobes, each pair symmetric with respect to a different axis. Manchado et al. have suggested that such nebulae have been formed by multiple shell ejections from a precessing rotating central star. For two cases, they propose that the precession of the rotation axis of the star is probably due to an external torque produced by a binary companion. Also an external torque due to a companion has been suggested by García-Segura (1997) to produce rotating jets through magnetic collimation around a precessing star. Note that Manchado et al. have ruled out the common envelope scenario as an explanation for quadrupolar morphology.

In N66, material ejections seem to have occurred over thousands of years, in bipolar episodic events along a precessing axis, which might indicate a binary scenario. The large dimensions of the twisted loops can be attributed to an old age. Taking a $V_{\exp }$ of about $100 \mathrm{~km} \mathrm{~s}^{-1}$, a kinematical age larger than 5000 years results for the edge of the loops, while the E-W lobes, with half the extension, would have been ejected 
Table 5. Physical conditions and chemical composition.

\begin{tabular}{|c|c|c|c|c|c|c|c|}
\hline \multirow[t]{2}{*}{ Parameter } & \multirow[t]{2}{*}{ Ion, line(s) } & \multirow[b]{2}{*}{ Pre-outb ${ }^{1}$} & \multirow[b]{2}{*}{ Approach $^{2}$} & \multicolumn{2}{|c|}{ - Value } & \multirow[b]{2}{*}{ Knot 3} & \multirow[b]{2}{*}{ Knot 2} \\
\hline & & & & Receding $^{2}$ & Knot 1 & & \\
\hline Flux ratio & [O III $]$ 4363/5007 & 0.0235 & 0.0275 & 0.0274 & 0.021 & 0.023 & 0.023 \\
\hline$"$ & [N II] 5755/6583 & 0.0227 & 0.0244 & 0.0245 & - & - & - \\
\hline$"$ & [S II] 4070/6725 & 0.289 : & 0.157 & 0.142 & - & - & - \\
\hline$”$ & [S II] 6731/6717 & 1.348 & 1.344 & 1.198 & 1.200 & - & 1.200 \\
\hline$"$ & [Ar IV] 4741/4713 & 0.827 & 0.905 & 0.832 & 1.000 & - & 0.833 \\
\hline$"$ & [O II] 3726/3729 & - & 1.500 & 1.368 & - & - & - \\
\hline$c(\mathrm{H} \beta)$ & & 0.16 & 0.23 & 0.27 & 0.13 & 0.20: & 0.15 \\
\hline$T_{\mathrm{e}}(\mathrm{K})$ & [O III] & 16460 & 17890 & 17870 & 15540 & 16080 & 16340 \\
\hline$T_{\mathrm{e}}(\mathrm{K})$ & [N II $]$ & 12220 & 12700 & 12890 & - & - & - \\
\hline$T_{\mathrm{e}}(\mathrm{K})$ & {$[\mathrm{S}$ II $]$} & - & 12680 & 14370 & - & - & - \\
\hline$n_{\mathrm{e}}\left(\mathrm{cm}^{-3}\right)$ & {$[\mathrm{S}$ II $]$} & 2030 & 2080 & 1410 & 1340 & (1300) & 1370 \\
\hline$n_{\mathrm{e}}\left(\mathrm{cm}^{-3}\right)$ & [Ar IV] & 1630 & 2850 & 1750 & 4000 & - & 1710 \\
\hline$n_{\mathrm{e}}\left(\mathrm{cm}^{-3}\right)$ & [O II] & - & 1770 & 1390 & - & - & - \\
\hline$n\left(\mathrm{He}^{0}\right) / n(\mathrm{H})$ & He I 5876 & $5.11 \mathrm{E}-2$ & $4.42 \mathrm{E}-2$ & $5.25 \mathrm{E}-2$ & $7.19 \mathrm{E}-2$ & - & 7.04E-2 \\
\hline$n\left(\mathrm{He}^{+}\right) / n(\mathrm{H})$ & He II 4686 & $6.81 \mathrm{E}-2$ & $6.94 \mathrm{E}-2$ & $6.04 \mathrm{E}-2$ & $5.10 \mathrm{E}-2$ & $7.27 \mathrm{E}-2$ & $5.80 \mathrm{E}-2$ \\
\hline$n\left(\mathrm{C}^{++}\right) / n(\mathrm{H})$ & [C III] 1909 & 7.77E-6 & - & - & $1.14 \mathrm{E}-5$ & $1.12 \mathrm{E}-5$ & $6.89 \mathrm{E}-6$ \\
\hline$n\left(\mathrm{C}^{3+}\right) / n(\mathrm{H})$ & [C IV] 1550 & $9.16 \mathrm{E}-6$ & - & - & $8.42 \mathrm{E}-6$ & $9.86 \mathrm{E}-6$ & 6.14E-6 \\
\hline$n\left(\mathrm{~N}^{+}\right) / n(\mathrm{H})$ & {$[\mathrm{N}$ II] 6584} & $1.29 \mathrm{E}-5$ & $1.48 \mathrm{E}-5$ & $1.22 \mathrm{E}-5$ & $8.72 \mathrm{E}-6$ & $5.34 \mathrm{E}-6$ & 8.07E-6 \\
\hline$n\left(\mathrm{~N}^{++}\right) / n(\mathrm{H})$ & [N III] 1750 & $3.20 \mathrm{E}-5$ & - & - & $4.08 \mathrm{E}-5$ & - & - \\
\hline$n\left(\mathrm{~N}^{3+}\right) / n(\mathrm{H})$ & [N IV] 1485 & $4.21 \mathrm{E}-5$ & - & - & $4.94 \mathrm{E}-5$ & $5.39 \mathrm{E}-5$ & $4.46 \mathrm{E}-5$ \\
\hline$n\left(\mathrm{~N}^{4+}\right) / n(\mathrm{H})$ & {$[\mathrm{N} \mathrm{v}] 1240$} & $4.58 \mathrm{E}-5$ & - & - & $2.60 \mathrm{E}-5$ & $3.45 \mathrm{E}-5$ & $3.77 \mathrm{E}-5$ \\
\hline$n\left(\mathrm{O}^{+}\right) / n(\mathrm{H})$ & [O II] 3727 & $1.60 \mathrm{E}-5$ & $1.71 \mathrm{E}-5$ & $1.35 \mathrm{E}-5$ & $9.13 \mathrm{E}-6$ & $4.34 \mathrm{E}-6$ & $6.01 \mathrm{E}-6$ \\
\hline$n\left(\mathrm{O}^{++}\right) / n(\mathrm{H})$ & [O III] 5007 & $7.60 \mathrm{E}-5$ & $6.18 \mathrm{E}-5$ & $6.12 \mathrm{E}-5$ & $9.65 \mathrm{E}-5$ & $7.11 \mathrm{E}-5$ & 8.07E-5 \\
\hline$n\left(\mathrm{O}^{3+}\right) / n(\mathrm{H})$ & [O IV] 1402 & $8.25 \mathrm{E}-5$ & - & - & $1.03 \mathrm{E}-4$ & $1.10 \mathrm{E}-4$ & - \\
\hline$n\left(\mathrm{Ne}^{++}\right) / n(\mathrm{H})$ & [Ne III] 3869 & $1.89 \mathrm{E}-5$ & $1.54 \mathrm{E}-5$ & $1.62 \mathrm{E}-5$ & $2.24 \mathrm{E}-5$ & $1.45 \mathrm{E}-5$ & $2.56 \mathrm{E}-5$ \\
\hline$n\left(\mathrm{Ne}^{3+}\right) / n(\mathrm{H})$ & [Ne IV] 4725 & $1.30 \mathrm{E}-5$ & - & - & $2.93 \mathrm{E}-5$ & - & - \\
\hline$n\left(\mathrm{Ne}^{3+}\right) / n(\mathrm{H})$ & [Ne IV] 2423 & $9.18 \mathrm{E}-6$ & - & - & $1.05 \mathrm{E}-5$ & $1.04 \mathrm{E}-5$ & $4.39 \mathrm{E}-6$ \\
\hline$n\left(\mathrm{Ne}^{4+}\right) / n(\mathrm{H})$ & {$[\mathrm{Ne} \mathrm{V}] 3423$} & $1.48 \mathrm{E}-5$ & $1.17 \mathrm{E}-5$ & $1.05 \mathrm{E}-5$ & $1.31 \mathrm{E}-5$ & $1.44 \mathrm{E}-5$ & $1.36 \mathrm{E}-5$ \\
\hline$n\left(\mathrm{~S}^{+}\right) / n(\mathrm{H})$ & [S II] 6725 & $2.12 \mathrm{E}-7$ & $3.08 \mathrm{E}-7$ & $2.82 \mathrm{E}-7$ & $1.21 \mathrm{E}-7$ & - & $1.17 \mathrm{E}-7$ \\
\hline$n\left(\mathrm{~S}^{++}\right) / n(\mathrm{H})$ & [S III] 6312 & $1.19 \mathrm{E}-6$ & $1.31 \mathrm{E}-6$ & $1.01 \mathrm{E}-6$ & $1.51 \mathrm{E}-6$ & - & - \\
\hline$n\left(\mathrm{Ar}^{++}\right) / n(\mathrm{H})$ & [Ar III] 7136 & $3.73 \mathrm{E}-7$ & $4.50 \mathrm{E}-7$ & $4.23 \mathrm{E}-7$ & - & - & - \\
\hline$n\left(\mathrm{Ar}^{3+}\right) / n(\mathrm{H})$ & [Ar IV] 4740 & $4.83 \mathrm{E}-7$ & $4.01 \mathrm{E}-7$ & $4.26 \mathrm{E}-7$ & $4.78 \mathrm{E}-7$ & - & $5.49 \mathrm{E}-7$ \\
\hline$n\left(\mathrm{Ar}^{4+}\right) / n(\mathrm{H})$ & [Arv] 7006 & $2.06 \mathrm{E}-7$ & - & - & - & - & - \\
\hline$n(\mathrm{He}) / n(\mathrm{H})$ & & $1.19 \mathrm{E}-1$ & $1.14 \mathrm{E}-1$ & $1.13 \mathrm{E}-1$ & $1.23 \mathrm{E}-1$ & $>7.27 \mathrm{E}-2$ & $1.28 \mathrm{E}-1$ \\
\hline$n(\mathrm{O}) / n(\mathrm{H})$ & corrected $^{3}$ & $1.62 \mathrm{E}-4$ & $1.48 \mathrm{E}-4$ & $1.24 \mathrm{E}-4$ & $1.51 \mathrm{E}-4$ & $>7.54 \mathrm{E}-5$ & $1.30 \mathrm{E}-4$ \\
\hline$n(\mathrm{~N}) / n(\mathrm{O})$ & $=n\left(\mathrm{~N}^{+}\right) / n\left(\mathrm{O}^{+}\right)$ & $8.06 \mathrm{E}-1$ & $8.64 \mathrm{E}-1$ & $9.03 \mathrm{E}-1$ & $9.55 \mathrm{E}-1$ & $1.23 \mathrm{E}+0$ & $1.34 \mathrm{E}+0$ \\
\hline$n(\mathrm{C}) / n(\mathrm{O})$ & $=n\left(\mathrm{C}^{++}\right) / n\left(\mathrm{O}^{++}\right)$ & $1.03 \mathrm{E}-1$ & - & - & 1.18E-1: & 1.57E-1: & $0.85 \mathrm{E}-1:$ \\
\hline$n(\mathrm{Ne}) / n(\mathrm{O})$ & $=n\left(\mathrm{Ne}^{++}\right) / n\left(\mathrm{O}^{++}\right)$ & $2.49 \mathrm{E}-1$ & $2.49 \mathrm{E}-1$ & $2.64 \mathrm{E}-1$ & $2.32 \mathrm{E}-1$ & $2.04 \mathrm{E}-1$ & $3.18 \mathrm{E}-1$ \\
\hline
\end{tabular}

2500 years ago, and knot 2 (which seems to be in the plane of the sky at a distance of $0.2^{\prime \prime}$ ) could be only 500 years old.

The analysis of the chemical composition of the nebular zones near the central shows that the nearest knots could contain He- and N-rich material. Undoubtedly the morphology, energetics, and chemical composition of N66 should be related to the extraordinary characteristics of its progenitor star, whose evolutionary status is still under debate. In order to explain the singular outburst and the odd chemical composition shown by the star, Hamann et al. (2003) have concluded that this object 
is probably a present close binary where a white dwarf accretes H-rich material from a non-degenerate secondary, possibly being on the way to become once a supernova of type Ia. The accreted matter would be continuously mixed and burned in the envelope of the white dwarf, producing the partly CNO-burnt composition observed in the [WN] stellar wind. However, it might happen that a larger amount of material accumulates in the WD surface. Then the stellar envelope would grow dramatically, and the star might eject accreted matter and add it to the nebula. The young nebular knots, showing high velocities and a slight enrichment of $\mathrm{He}$ and $\mathrm{N}$, might have been expelled in such events, which should have been more dramatic than the outburst observed in the past decade.

Acknowledgements. This work received partial support from DGAPA/UNAM (grant IN114601) and CONACYT/México (grant 34594-E). M.T.R. received partial support from FONDAP/Chile (\#15010003), a Guggenheim Fellowship and Fondecyt/Chile (\#1010404).

\section{References}

Benedict, G. F., McArthur, B. E., Fredrick, L. W., et al. 2002, AJ, 124, 1695

Blades, J. C., Barlow, M. J., Albrecht, R., et al. 1992, ApJ, 398, L41

Dopita, M. A., Ford, H. C., Lawrence, C. J., \& Webster, B. L. 1985, ApJ, 296, 390
Dopita, M. A., Ford, H. C., Bohlin, R., Evans, I. N., \& Meatheringham, S. J. 1993, ApJ, 418, 804

D’Odorico, S., Cristiani, S., Dekker, H., et al. 2000, Proc. SPIE, 4005, 121

Feast, M. W. 1988, in The Extragalactic Distance Scale, ed. S. van den Bergh, \& C. J. Pritchet, ASP Conf. Ser., 4, 4

García-Segura, G. 1997, ApJ, 489, L192

García-Segura, G., \& López, J. A. 2000, ApJ, 544, 336

Hamann, W.-R., Peña, M., Gräfener, G., \& Ruiz, M. T. 2003, A\&A, 409, 969

Kingsburgh, R. L., \& Barlow, M. J. 1994, MNRAS, 271, 257

López, J. A., Meaburn, J., Bryce, M., \& Holloway, J. 1998, ApJ, 493, 803

Manchado, A., Stanghellini, L., \& Guerrero, M. 1996, ApJ, 466, L95

Morgan, D. H., Parker, Q. A., \& Cohen, M. 2003, MNRAS, 346, 719

Peña, M., Torres-Peimbert, S., Peimbert, M., Ruiz, M. T., \& Maza, J. 1994, ApJ, 428, L9

Peña, M., Peimbert, M., Torres-Peimbert, S., Ruiz, M. T., \& Maza, J. 1995, ApJ, 441, 343

Peña, M., Hamann, W.-R., Koesterke, L., et al. 1997, ApJ, 491, 233

Pottasch, S. R., Baud, B., Beintema, D., et al. 1984, A\&A, 138, 10

Schwering, P. B. W. 1989, A\&AS, 79, 105

Seaton, M. 1979, MNRAS, 187, 73

Stanghellini, L., Shaw, R. A., Mutchler, M., et al. 2002, ApJ, 575, 178

Storey, P. I., \& Hummer, D. G. 1995, MNRAS, 272, 41

Shaw, R. A., Stanghellini, L., Villaver, E., et al. in prep.

Soker, N. 2002, ApJ, 568, 726

Vassiliadis, E. 1996, ApJ, 465, 748

Vassiliadis, E., Dopita, M. A., Meatheringham, H. C., et al. 1998, ApJ, 503,253 